\title{
Pharyngostomy tubes for gastric conduit decompression
}

\author{
Natasha M. Rueth, MD, ${ }^{a}$ Natasha Lee, ${ }^{a}$ Shawn S. Groth, MD, ${ }^{a}$ Sarah C. Stranberg, MA, CCC-SLP, \\ Michael A. Maddaus, MD, ${ }^{a}$ Jonathan D'Cunha, MD, PhD, and Rafael S. Andrade, MD ${ }^{a, c}$
}

Objective: This article illustrates our operative technique for pharyngostomy tube placement and describes our clinical experience with pharyngostomy use for gastric conduit decompression after esophagectomy.

\begin{abstract}
Methods: We retrospectively reviewed patients undergoing pharyngostomy tube placement for gastric conduit decompression after esophagectomy from January 2008 to August 2009. Patients were included if they had a pharyngostomy tube placed at esophagectomy (prophylactic placement) or as a means of decompression after postesophagectomy anastomotic leak (therapeutic placement). We collected operative and clinical data and performed a descriptive statistical analysis.
\end{abstract}

Results: We placed 25 pharyngostomy tubes for gastric conduit decompression after esophagectomy. Eleven were placed prophylactically (44\%); the remaining 14 were placed therapeutically $(56 \%)$ after anastomotic leak. Prophylactic pharyngostomy tubes remained in place a median of 8 days (range 4-17 days), whereas therapeutic pharyngostomy tubes were left in place a median of 15 days (range 7-125 days). There were 4 infectious complications $(16 \%)$ unrelated to length of pharyngostomy use: 2 cases of cellulitis (resolved with antibiotics, tube remaining in place) and 2 superficial abscesses after tube removal requiring bedside débridement. Seventy-two percent of patients underwent swallow evaluation; $22 \%$ of these patients had radiographic evidence of aspiration.

Conclusions: Pharyngostomy tube placement for gastric conduit decompression after esophagectomy is simple, and tubes can stay in place for prolonged periods. Our experience suggests that pharyngostomy tubes are a safe alternative to nasogastric drainage. (J Thorac Cardiovasc Surg 2010;140:373-6)

Gastric conduit decompression after esophagectomy, which is important to minimize distention and ischemia, is generally accomplished with nasogastric tube (NGT) drainage for varying periods (3-7 days). Not only are NGTs uncomfortable, however, they contribute their own morbidity to the procedure, including sinusitis, pharyngitis, and aspiration events. $^{1,2}$

A pharyngostomy tube (PT), placed percutaneously through the lateral pharyngeal wall, is a safe but uncommonly used alternative to the NGT for gastric conduit decompression. ${ }^{1-3}$ A PT offers certain potential advantages relative to the conventional NGT, including greater patient comfort, less interference with pulmonary toilet and patient mobility, and the possibility of prolonged use (several months if necessary). In this study, we provide a detailed technical illustration of PT placement and briefly describe our clinical experience with PTs in esophagectomy patients.

\footnotetext{
From the Department of Surgery, ${ }^{\mathrm{a}}$ Division of General Thoracic and Foregut Surgery, University of Minnesota, Rehabilitation Services, ${ }^{\mathrm{b}}$ University of Minnesota Medical Center, Fairview; and the Veterans Administration Medical Center, ${ }^{\mathrm{c}}$ Minneapolis, Minn.

Disclosures: None.

Received for publication Oct 17, 2009; revisions received Jan 25, 2010; accepted for publication Feb 26, 2010; available ahead of print April 15, 2010.

Address for reprints: Rafael S. Andrade, MD, MMC195, 420 Delaware Street SE,

Minneapolis, MN 55455 (E-mail: andr0119@umn.edu).

$0022-5223 / \$ 36.00$

Copyright (C) 2010 by The American Association for Thoracic Surgery

doi:10.1016/j.jtcvs.2010.02.043
}

\section{MATERIALS AND METHODS}

After approval by the institutional review boards of both institutions, we performed a systematic, retrospective review of our surgical patient database at the University of Minnesota Medical Center and the Minneapolis Veterans Administration Medical Center. We included all patients undergoing PT placement for gastric decompression after esophagectomy between January 2007 and August 2009. Information was collected on patient demographic characteristics, surgical indications, type of esophagectomy, postoperative course, and surgical and upper aerodigestive functional outcomes.

Prophylactic PT placement was defined surgical PT placement at the time of original esophagectomy for gastric conduit decompression; patient selection for prophylactic PT placement was surgeon dependent. Therapeutic PT use was defined as PT placement to decompress the gastric conduit as an adjunct to the management of an anastomotic leak after esophagectomy; this is a routine step in managing anastomotic leaks at our institutions. We evaluated upper aerodigestive function radiographically and in conjunction with a certified speech language pathologist (S.S.). Descriptive statistics are reported as medians with ranges.

\section{PT Placement: Procedure Description}

PT placement is performed with the patient in supine position under general anesthesia.

(1) Identification of posterior tonsillar pillar (palatopharyngeal arch). We use a laryngoscope to see the posterior tonsillar pillar. Under direct visualization, we place a long, curved clamp just beyond it and direct the tip laterally (Figure 1).

(2) Determination of incision site. Once the tip of the clamp is against the lateral pharyngeal wall, we can readily palpate it through the skin, about $1 \mathrm{~cm}$ below the mandible and 1 to $2 \mathrm{~cm}$ anterior to the mandibular angle (Figure 2, A). This anatomic point is pivotal to avoid inadvertent damage to the superior laryngeal artery, vein, submandibular gland, and carotid artery. When properly positioned, only subcutaneous tissue separates the instrument from the cutaneous exit site. 


\section{Abbreviations and Acronyms \\ NGT = nasogastric tube \\ PT = pharyngostomy tube}

(3) Percutaneous placement of PT. We make a 5-mm skin incision over the tip of the clamp; the clamp can then be advanced bluntly-without spreading, to avoid unnecessary dilation of the tract through the soft tissues (usually $5-10 \mathrm{~mm}$ in thickness). A $16 \mathrm{~F}$ to $18 \mathrm{~F}$ PT (any type of tube traditionally used for nasogastric decompression) is then pulled into the oropharynx with the clamp (Figure 2, $B$ ) and advanced into the gastric conduit. We place the tip of the tube 2 to $3 \mathrm{~cm}$ past the desired position in the conduit and then pull it back at the skin to avoid curling in the pharynx. Superficial bleeding from the tract is generally tamponaded by the PT; alternatively, a stitch can be placed to obtain hemostasis if needed.

(4) Routine management. The tube is placed to low suction drainage $(25 \mathrm{~mm} \mathrm{Hg})$ and is removed bedside at the discretion of the operating surgeon. The cutaneous fistula is covered and allowed to heal spontaneously.

\section{RESULTS}

During the specified period, we placed a total of 25 PTs for gastric conduit decompression after esophagectomy. During the same period, we performed a total of 34 esophagectomies at our institution. Of the PTs placed, 11 were placed prophylactically at the time of original operation (prophylactic PTs, 44\%), whereas 14 were placed for therapeutic purposes in the postoperative setting, always after an anastomotic leak (therapeutic PTs, 56\%). Nearly half of patients in the prophylactic group $(n=5,45.5 \%)$ received preoperative neoadjuvant therapy.

Of the 25 esophageal resections performed, $20(80 \%)$ were performed at our institution (8 totally minimally invasive with intrathoracic anastomosis, 6 in open Ivor Lewis fashion, and 6 hybrid procedures of thoracoscopy followed by open, transhiatal resections). Sixteen of these 20 patients underwent simultaneous pyloric opening procedures (14 pyloroplasty or pyloromyotomy, 2 pyloric onabotulinumtoxin A [Botox, INN botulinum toxin type A] injections), and 1 patient underwent total gastrectomy with intrathoracic esophagojejunostomy. The remaining 5 esophagectomies were performed at outside institutions with subsequent referral to our hospital for management of complicated anastomotic leaks.

Prophylactic PTs remained in place a median of 8 days (range 4-17 days), whereas therapeutic PTs were retained for a median of 15 days (range 7-125 days). Table 1 summarizes pertinent hospital data.

There were no instances of accidental PT removal or dislodgment, and only 1 patient had the PT removed prematurely because of posterior pharyngeal pain (notably, this patient did not tolerate the NGT either, for the same reason). We found 4 infectious complications $(16 \%), 2$ of which were cases of cellulitis that were resolved with intravenous
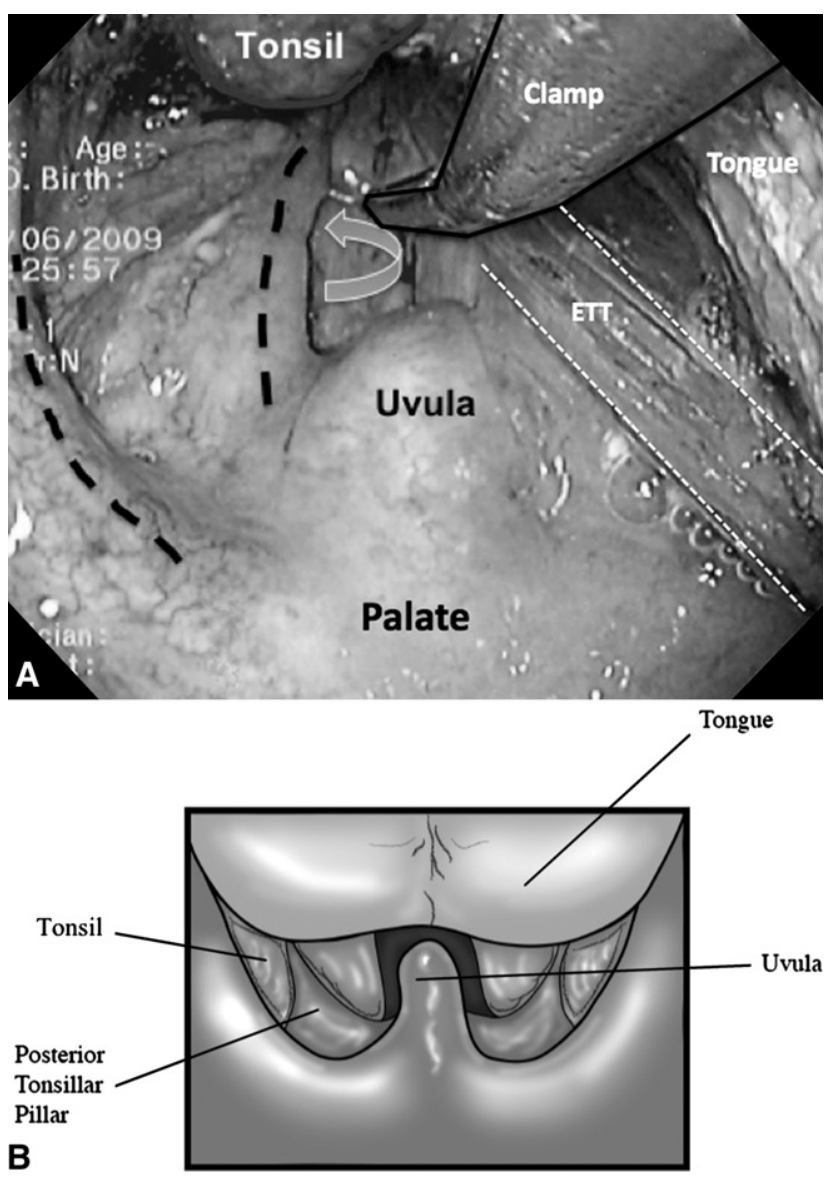

FIGURE 1. A, Operator view of left oropharynx (patient supine) with tongue and endotracheal tube (ETT) displaced to right. Forceps in oropharynx demonstrate fossa (arrow) distal to left posterior tonsillar pillar (dashed lines). B, Illustration of anatomy from photograph.

antibiotics while the PT was still in place. The remaining 2 patients had superficial abscesses develop after PT removal, necessitating open drainage at bedside. One death $(4 \%)$ occurred, from an aortogastric fistula resulting from an intrathoracic leak; this death was unrelated to either placement or management of the PT.

In total, 32 radiographic aerodigestive functional studies (17 esophagrams, 15 modified barium swallow studies) were performed on 18 patients ( $72 \%$ of the population). The overall aspiration rate among studied patients with the PT in place was $22 \% ; 1$ patient $(4 \%)$ with radiographic evidence of aspiration with the PT in place had subsequent aspiration pneumonia and respiratory failure requiring brief ventilatory support. The remaining 3 patients with radiographically confirmed aspiration events had no associated respiratory complications.

\section{DISCUSSION}

In this study, we have illustrated our operative technique for PT placement and described our clinical experience with its use for gastric conduit decompression after esophagectomy. 

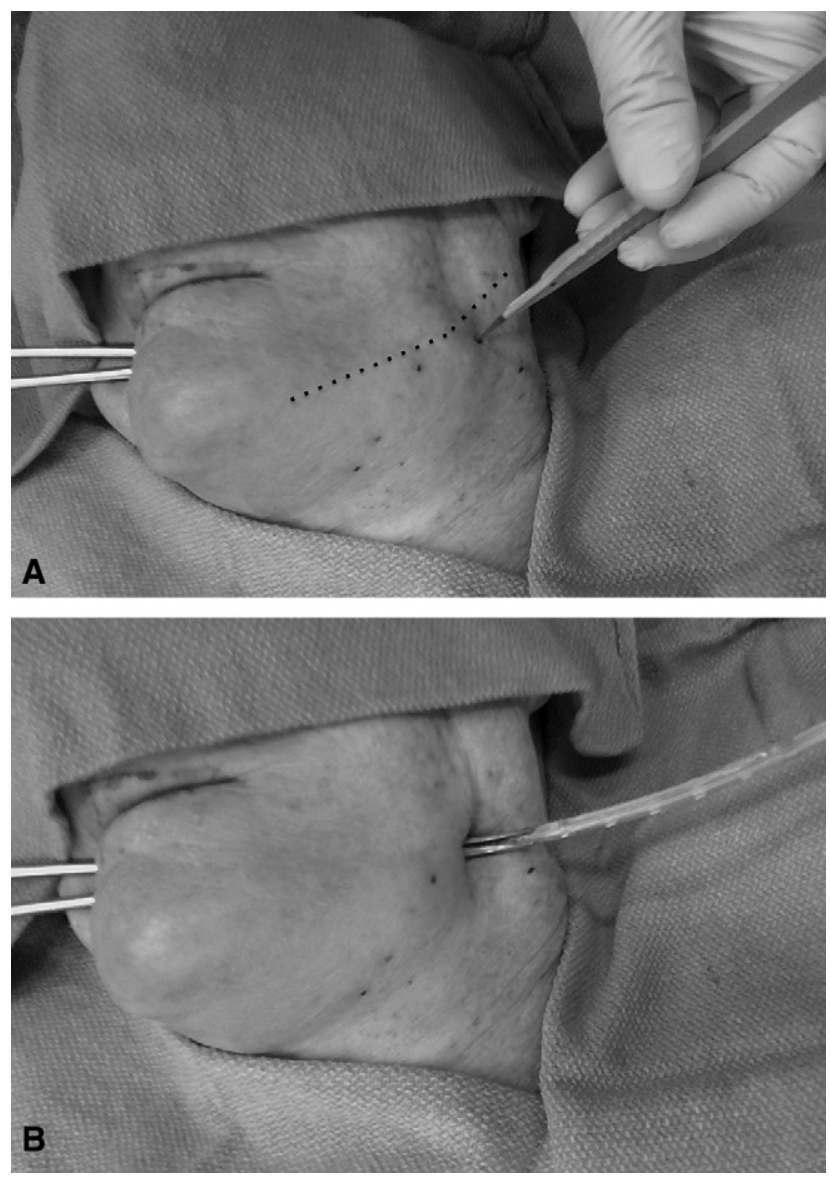

FIGURE 2. A, Exterior anatomy for pharyngostomy tube placement. Scalpel marks exit site for pharyngostomy tube; dashed line indicates angle of mandible. B, Percutaneous placement of pharyngostomy tube. Flexible tube is pulled into mouth through exit site.

PTs have been used for gastric decompression and enteral feeding after head and neck surgery ${ }^{4,5}$; recently, 2 reports have attempted to revitalize this procedure as an adjunct to foregut surgery. ${ }^{1,3}$ The interest in PTs among thoracic surgeons has emerged from the need for an alternative to NGTs for prolonged ( $>5$ days) gastric decompression.

TABLE 1. Hospital data for prophylactic versus therapeutic pharyngostomy tube placement

\begin{tabular}{lcc}
\hline & $\begin{array}{c}\text { Prophylactic } \\
\text { PT }(\mathbf{n}=\mathbf{1 1})\end{array}$ & $\begin{array}{c}\text { Therapeutic } \\
\text { PT }(\mathbf{n}=\mathbf{1 4})\end{array}$ \\
\hline $\begin{array}{l}\text { Hospital stay (d, median and range) } \\
\text { Surgical intensive care stay }\end{array}$ & $16(11-23)$ & $20(8-125)$ \\
$\quad(d$, median and range) & $3(2-13)$ & $10(1-125)$ \\
$\begin{array}{l}\text { Time to PT removal } \\
\quad(d, \text { median and range) }\end{array}$ & $8(4-7)$ & $15(7-125)$ \\
Pneumonia (no.) & $2(18.2 \%)$ & $5(35.7 \%)$ \\
Anastomotic leak (no.) & $1(9.1 \%)$ & $14(100 \%)$ \\
PT complications (no.) & $3(27.3 \%)$ & $1(7.1 \%)$ \\
\hline
\end{tabular}

$P T$, Pharyngostomy tube
Routine postoperative decompression of the upper gastrointestinal tract has long been the standard of care. ${ }^{1-3}$ After esophagectomy, theoretic advantages include protection against aspiration and decreased risk of anastomotic leak from distention-induced gastric conduit ischemia. ${ }^{6,7} \mathrm{NGT}$ decompression is the mainstay of therapy for patients requiring decompression of the gastrointestinal tract; however, NGT decompression itself may provide an additional source of complications, including aspiration, associated pulmonary compromise, patient discomfort, and sinusitis. ${ }^{1,8}$ In a recent systematic review including more than 4000 patients, Nelson and colleagues ${ }^{7}$ reported that among those with upper gastrointestinal tract surgery, pulmonary complications were more frequent when routine NGT decompression was used.

Schuchert and colleagues ${ }^{3}$ recently published a report on a series of 145 patients undergoing open esophagectomy and transcervical gastric tube drainage (placed through a separate proximal cervical esophagostomy and secured with a pursestring suture, necessitating additional esophageal manipulation), with minimal morbidity attributed to tube use. ${ }^{1}$ In their series, cellulitis was the only reported complication $(2.9 \%)$; esophagostomy tubes were left in place for a mean of 8 days. From the same institution, Kent and colleagues ${ }^{3}$ reported on a heterogeneous group of 38 patients with PTs placed postoperatively for a multitude of purposes, including mediastinal abscess drainage, enteral nutrition, and malignant bowel obstruction; they reported a tube migration rate of $23.7 \%$.

Our detailed technical description and clinical results further the PT experience, represented previously by only a single institution, and demonstrate that PTs can be used for gastric decompression regardless of anastomosis location. Our technique of PT placement is simple, and as yet we have not had any intraoperative complications or unexpected postoperative tube migration or dislodgment. We emphasize 2 key principles for safe placement: (1) clear identification of the posterior tonsillar pillar, and (2) easy palpation of the tip of the clamp on the skin surface. Bleeding from the tract generally stops as a result of tamponade once the PT is in place. Alternatively, a stitch can be placed to obtain hemostasis if needed.

We observed an infectious complication rate of $16 \%$. Cellulitis can be treated with antibiotics with the PT in place. In 2 patients, abscesses developing after PT removal required open bedside drainage, with no long-term sequelae. An overall $16 \%$ incidence of surgical site infection argues for proper patient selection to avoid adding complications in low-risk postesophagectomy patients who may need only brief (2-3 days) gastric decompression.

Objective proof of upper aerodigestive dysfunction was evident in $22 \%$ of the 18 patients tested ( $72 \%$ of the population underwent testing). One patient with upper aerodigestive dysfunction and a tracheostomy in place had pneumonia develop; the remaining 3 patients with documented aspiration 
did not have respiratory complications. The rate of clinically evident aspiration in this study is not different from the published incidence of aerodigestive dysfunction after esophagectomy $(20 \%),{ }^{9,10}$ suggesting that PTs do not add upper aerodigestive morbidity to that of esophagectomy alone.

In our experience, as well as in the recent reports, ${ }^{1}$ PT use for gastric decompression after esophagectomy is well tolerated by patients and allows easy mobilization, although we have not used a specific questionnaire to evaluate patient comfort. Without control data, we cannot make an objective comparative statement regarding NGT decompression. We do prefer PTs to NGTs for prolonged ( $>5$ days) gastric decompression, however, because PTs do not appear to be associated with more complications than NGTs, are probably more comfortable for patients, and rarely become accidentally dislodged.

Although we have not defined absolute selection criteria, our current practice is to place PTs prophylactically in older patients (>70 years), current smokers, patients who have received neoadjuvant therapy, and those with certain high-risk medical comorbidities (eg, heart disease, lung disease, obesity, diabetes, malnourishment). Studies have documented that several of these risk factors are independent predictors of postesophagectomy morbidity. ${ }^{11-13}$ Additionally, we now place a therapeutic PT in all patients with anastomotic leaks, because prolonged conduit decompression is required in such cases. To date, our only absolute contraindication to PT placement has been previous neck radiation, because of the potential for development of a chronic pharyngocutaneous fistula.

Our experience leads us to conclude that that PT use is a safe and valuable adjunct for prolonged ( $>5$ days) gastric decompression after esophagectomy in appropriately selected patients. We have provided this detailed description of our operative technique for PT placement and summary of our clinical experience with its use to guide esophageal surgeons interested in adopting this clinical tool in their own practices.

We thank Lisa D'Cunha for original artwork.

\section{References}

1. Schuchert MJ, Pettiford BL, Landreneau JP, Waxman J, Kilic A, Santos RS, et al. Transcervical gastric tube drainage facilitates patient mobility and reduces the risk of pulmonary complications after esophagectomy. J Gastrointest Surg. 2008;12: 1479-84.

2. Barros JL. Cervical gastrostomy in the surgical treatment of oesophageal cancer by reconstruction with the stomach. Thorax. 1981;36:863-5.

3. Kent MS, Awais O, Schuchert MJ, Adusumilli PS, Keeley S, Alvelo-Rivera M, et al. Cervical pharyngostomy: an old technique revisited. Ann Surg. 2008;248: 199-204.

4. Graham WP 3rd, Royster HP. Simplified cervical esophagostomy for long term extraoral feeding. Surg Gynecol Obstet. 1967;125:127-8.

5. Lyons JH Jr. Cervical pharyngostomy. A safe alternative for gastrointestinal decompression. Am J Surg. 1974;127:387-91.

6. Nelson R, Edwards S, Tse B. Prophylactic nasogastric decompression after abdominal surgery. Cochrane Database Syst Rev. 2007;(3):CD004929.

7. Nelson R, Tse B, Edwards S. Systematic review of prophylactic nasogastric decompression after abdominal operations. Br J Surg. 2005;92:673-80.

8. Rouby JJ, Laurent P, Gosnach M, Cambau E, Lamas G, Zouaoui A, et al. Risk factors and clinical relevance of nosocomial maxillary sinusitis in the critically ill. Am J Respir Crit Care Med. 1994;150:776-83.

9. Leder SB, Bayar S, Sasaki CT, Salem RR. Fiberoptic endoscopic evaluation of swallowing in assessing aspiration after transhiatal esophagectomy. J Am Coll Surg. 2007;205:581-5

10. Keeling WB, Lewis V, Blazick E, Maxey TS, Garrett JR, Sommers KE. Routine evaluation for aspiration after thoracotomy for pulmonary resection. Ann Thorac Surg. 2007;83:193-6.

11. Atkins BZ, Shah AS, Hutcheson KA, Mangum JH, Pappas TN, Harpole DH Jr, et al. Reducing hospital morbidity and mortality following esophagectomy. Ann Thorac Surg. 2004;78:1170-6.

12. Bartels H, Stein HJ, Siewert JR. Preoperative risk analysis and postoperative mortality of oesophagectomy for resectable oesophageal cancer. Br J Surg. 1998;85: $840-4$.

13. Bauer JJ, Gelernt IM, Salky BA, Kreel I. Is routine postoperative nasogastric decompression really necessary? Ann Surg. 1985;201:233-6. 